\title{
Three dimensional imaging of arc of Buhler
}

\author{
Kemal Arda ${ }^{*}$ and Halil Ibrahim ${ }^{2}$ \\ ${ }^{1}$ Ankara Atatürk Research and Training Hospital, Department of Radiology, Ankara, Turkey \\ ${ }^{2}$ Bozok University Faculty of Medicine Department of Radiology, Yozgat, Turkey
}

\begin{abstract}
The Arc of Buhler (AOB) is one of the variants and anastomoses, between Celiak trunkus (CelT) and Superior mesenteric artery (SMA).

AOB is a persistent embryonic ventral anastomosis. In 74- year- old man, AOB was diagnosed by using MDCT. We present a case report and discuss AOB and collateral pathways between CelT and SMA.
\end{abstract}

\section{Introduction}

The gastrointestinal system is mainly supplied by celiac trunk (CelT), superior mesenteric artery (SMA), and inferior mesenteric artery [1]. The celiac trunk is a large arterial vessel of the upper abdomen which arises below the aortic hiatus at the level of $\mathrm{T} 12^{\text {th }}$ the vertebral body. It is divided into three main branches: common hepatic, splenic and left gastric arteries [2]. The SMA arises from the anterior aorta near the L1-2 level, 1-2 cm below the celiac artery origin [2]. The SMA branches are inferior anterior and posterior pancreaticoduodenal arteries, middle and right colic arteries, iliocolic artery, and jejunal and ileal branches [3].

Numerous anatomical variants and anastomoses have been reported between these arteries [1]. The Arc of Buhler (AOB), which is one of these variants and anastomoses, is a persistent embryonic ventral anastomosis between the CelT and the SMA [1,5]. It was first described in 1904 by Buhler [1,4]. The mesenteric vascular collaterals and variants are of utmost importance to explain of symptoms, gaining a better understanding of pathologies, and planning the interventions $[1,5]$. Herein, we present an interesting case of $\mathrm{AOB}$ with multiple detector computed tomography (MDCT) images.

\section{Case report}

A 74-year-old man was admitted to our hospital with progressive abdominal and back pain for two months. He lost $5 \mathrm{~kg}$ during this period. His past clinical history was nonspecific. He had no abdominal surgical history. His family history was unremarkable for a malignancy. On physical examination, there were no remarkable findings. The biochemical tests and electrocardiogram showed no specific alterations. Upper gastrointestinal endoscopy which was done for abdominal pain revealed antral gastritis.

Abdominal ultrasonography (USG) revealed tortuous vessels near aorta and SMA. The CelT was unable to be visualized by USG. Then, multiple detector computed tomography (MDCT) angiography was performed to visualize the aortic branches and the tortuous vessels. Multi-planar and maximum intensity projection (MIP) reconstructions showed celiac trunk abnormality. The MDCT showed AOB between the SMA and CelT (Figures 1-3). However, we were unable to visualize the celiac trunk originating from the aorta and a possible obstruction of the main arteries. All vessels were in normal calibration and contrast enhancement was normal. The epigastric pain was suspected to be due to the antral gastritis and medical treatment was given. The complaints gradually resolved with medical treatment. A written informed consent was obtained from the patient.

\section{Discussion}

The celiac artery, superior and inferior mesenteric arteries are three main ventral branches of the abdominal aorta which are arterial supplies of digestive organs of the upper abdomen $[1,2]$. The anatomical variations of the celiac trunk, SMA, and their branches are often caused by the developmental changes in the ventral splanchnic arteries [1]. These anatomical variations have been described by several authors [2-4].

The mesenteric arterial circulation is characterized by multiple collateral branches [2]. These collaterals protect the bowel from potential infarction and ischemia [2].

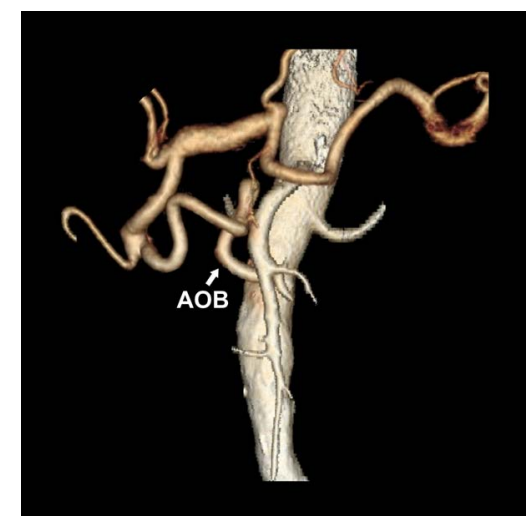

Figure 1. Arc of Buhler was demonstrated in 3D reconstruction of MDCT images.

Correspondence to: Kemal Arda, Ankara Atatürk Research and Training Hospital, Department of Radiology, Ankara, Turkey, Tel: + 90- 5067843044; Fax: + 90- 312 5932936; E-mail: drkemalarda@gmail.com

Key words: celiac trunk, superior mesenteric artery, arc of Buhler, variation

Received: June 30, 2016; Accepted: July 20, 2016; Published: July 25, 2016 


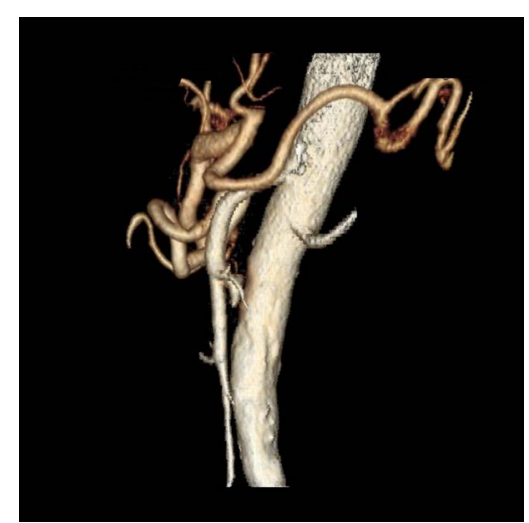

Figure 2. Sagittal view of the same artery.

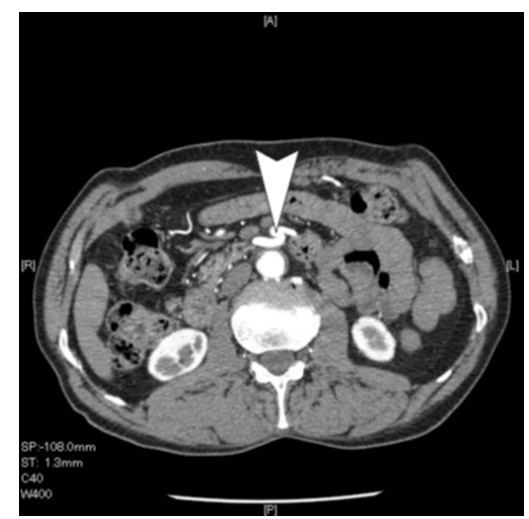

Figure 3. The connection between Celiactruncus and aorta could not be seen in the axial image.

Furthermore, there are three main primary collateral pathways for CelT and SMA. These are gastro duodenal artery, pancreoticoduodenal arteries, and dorsal pancreatic artery [2,5]. Additionally, there are two collateral pathways which are persistently embryologic origin, namely the $\mathrm{AOB}$ and arc of Barkow [2,5].

The AOB, which is rare is defined by the failure of regression of ventral segmental anastomoses between the segmental arteries $\left(10^{\text {th }}\right.$ $13^{\text {th }}$ ) during the embryonic life $[4,5]$. Its incidence ranges from 1 to $4 \%$ $[1,4,5]$. There are several studies reporting aneurysms of the AOB $[5,6]$.

In our case, the patient with abdominal pain was examined by USG initially. Although the CelT was unable to be visualized by USG, we detected tortuous vessels near the aorta and SMA. Therefore the
MDCT examination and MDCT angiography were performed to demonstrate the aortic branches and the tortuous vessels. However, we were unable to detect a direct conjunction between the aorta and CelT. Also, there was no obstruction of the CelT or a trace of the CelT in the aorta (i.e., sacculation, etc.). Subsequently, the MDCT axial images were reconstructed as three-dimensional and we detected an anastomosis between the SMA and gastroduodenal artery, a branch of the CelT, indicating an AOB. Multi-planar and MIP reconstructions also showed celiac trunk abnormality. Nonetheless, we were unable to visualize the celiac trunk originating from the aorta and a possible obstruction of the main arteries.

In a case series, Berteli et al. [7] reported three cases of anastomotic arterial trunk between the celiac artery and SMA ( $0.4 \%$ in vivo). The MDCT and multi-planar and MIP reconstructions offers a high quality angiograms to visualize such anomalies [5]. Similarly, we detected a variation of the CelT and SMA using MDCT angiography in our case.

In conclusion, the different forms of these variations should be considered during both surgical and non-surgical examinations. The radiologist should be aware the possibilities of arterial variations. Arterial variations such as AOB should keep in mind during abdominal surgeries. Therefore, many complications can be prevented.

\section{Consent}

A written informed consent was obtained from the patient for the publication of this case report.

\section{References}

1. Ochoa JE, Pointer DT Jr, Hamner JB (2016) Vascular Anomalies in Pancreaticoduodenectomy: A Lesson Learned. Case Rep Surg 2016: 5792980. [Crossref]

2. Walker TG (2009) Mesenteric vasculature and collateral pathways. Semin Interven Radiol 26: 167-174.[Crossref]

3. De Martino RR (2015) Normal and variant mesenteric anatomy. In Mesenteric vascular disease (1stedn), New York, Springer 9-23.

4. Rusu MC, Jianu AM, Sztika D, Cuzino D, Loreto C (2011) Three extremely rare anatomic variants of the hepatic artery. Ann Vasc Surg 25: 1138.[Crossref]

5. Kageyama Y, Kokudo T, Amikura K, Miyazaki Y, Takahashi A, et al. (2016) The arc of Buhler: special considerations when performing pancreaticoduodenectomy. Surg Case Rep 2: 21.[Crossref]

6. Jayia P, Hosney S, Subramanian A, Onnie C (2011) Arc of Buhler aneurysm: a rare cause of obstructive jaundice. Vasc Endovascular Surg 45: 92.[Crossref]

7. Bertelli E, Di Gregorio F, Civeli L (1991) [Various cases of direct connections between the celiac artery and the superior mesenteric]. Arch Ital Anat Embriol 96: 281-289. [Crossref]

Copyright: (C2016 Arda K. This is an open-access article distributed under the terms of the Creative Commons Attribution License, which permits unrestricted use, distribution, and reproduction in any medium, provided the original author and source are credited. 